\title{
Combined therapy against recurrent and intracranial invasion of sinonasal hemangiopericytoma: A case report
}

\author{
XIAOHONG WANG ${ }^{1}$, JIANGONG WANG ${ }^{1}$, WANNING HU ${ }^{2}$ LEI WANG $^{3}$ and YUFENG LI $^{2}$ \\ ${ }^{1}$ Department of Medical Oncology; ${ }^{2}$ The Cancer Institute; ${ }^{3}$ Department of Pathology, Tangshan People's Hospital, \\ Tangshan, Hebei 063001, P.R. China
}

Received July 15, 2014; Accepted March 12, 2015

DOI: $10.3892 / \mathrm{ol} .2015 .3236$

\begin{abstract}
Sinonasal hemangiopericytoma (HPC) is a soft tissue tumor of vascular origin. Open surgical methods and endoscopic techniques are considered the standard treatments for sinonasal HPC. However, local recurrences resulting from residual tumors are common. Adjuvant radiotherapy and chemotherapy have also been used to treat HPC, however, the number of studies which have investigated effective adjuvant treatments in the literature are limited. The current study reports a 42-year-old male with recurrent and intracranial invasion of sinonasal HPC, diagnosed in Xuanwu Hospital (Beijing, China). The patient underwent multiple surgeries to remove the tumors, however, no adjuvant therapy was adopted during this period and the tumors reoccurred within 1 year. On admittance to Tangshan People's Hospital (Tangshan, China), the patient presented with limited mouth opening and chewing ability, and hearing loss. Under observation using an electron microscope, HPC usually consists of spindle-shaped cells with elongated nuclei and displays characteristic staghorn-like vascular channels. In the present case, immunohistochemical studies were performed on paraffin-embedded sections of the tumor. The tumor cells expressed CD34, CD68(+/-), epithelial membrane antigen, CD31, $\alpha$-actin, desmin, CD99, S-100, B-cell lymphoma-2 and Ki-67 (30\%), but were negative for creatine kinase. The patient was treated with intensity-modulated radiotherapy and adjuvant chemotherapy, which demonstrated efficacy. No recurrence and metastasis was observed at the 1 year follow-up subsequent to the combined therapy.
\end{abstract}

\section{Introduction}

Hemangiopericytoma (HPC) is a soft tissue tumor of vascular origin and can originate from anywhere in the

Correspondence to: Ms. Xiaohong Wang, Department of Medical Oncology, Tangshan People's Hospital, 65 Sheng-Li Road, Tangshan, Hebei 063001, P.R. China

E-mail: wangxiaohongts@126.com

Key words: sinonasal, hemangiopericytoma, radiotherapy, chemotherapy human body. HPC cases that arise in the head and neck account for $\sim 15-25 \%$ of cases, $5 \%$ of which develop in the nasal and sinus area $(1,2)$. Sinonasal HPC accounts for $\leq 1 \%$ of sinonasal tumors, which often reoccur locally but rarely metastasize (3). Surgery is the main treatment method for primary and recurrent sinonasal HPC (4). Open surgical methods and endoscopic techniques have been considered as standard care of sinonasal HPC. Surgical removal resulted in no recurrence in $79.7 \%$ of the cases (4). Due to the complexity of nasal anatomy it is difficult to completely excise the HPC lesion by open or endoscopic surgery. The residual tumor is a major cause of local recurrence of sinonasal HPC, therefore, post-surgical rehabilitation for HPC remains a challenge. Only two cases have previously reported the use of adjuvant radiotherapy for residual or recurrent lesions and very few reports of chemotherapy for sinonasal HPC have been recorded $(1,5)$. The present case study reports that adjuvant radiotherapy and chemotherapy were effective to control the recurrent and intracranial invasion of one case of sinonasal HPC.

\section{Case report}

Patient presentation. In October 2011, a 42-year-old man diagnosed with recurrent and intracranial invasion of sinonasal HPC, was admitted to Xuanwu Hospital. The patient underwent multiple surgeries to remove the tumors, however, no adjuvant therapy was adopted during this period and the tumors reoccurred within 1 year. In December 2012, on admittance to Tangshan People's Hospital (Tangshan, China), the patient presented with limited mouth opening and chewing ability, and hearing loss. Maxillary sinus puncture was performed and the biopsy specimens were fixed with $10 \%$ formalin.

Pathological examination. The formalin-fixed, paraffin-embedded tissue sections ( $4 \mu \mathrm{m}$ thick) were deparaffinized in xylene and dehydrated through a graduated alcohol series into water. The sections were then used for hematoxylin and eosin (HE) staining or immunohistochemical analysis. For HE staining, briefly, the sections were incubated in working solution of Mayer's hematoxylin for $10 \mathrm{~min}$ (stain nuclear blue) and then rinsed in water followed by counterstain in eosin-phloxine solution for $1 \mathrm{~min}$ (stain cyto- 

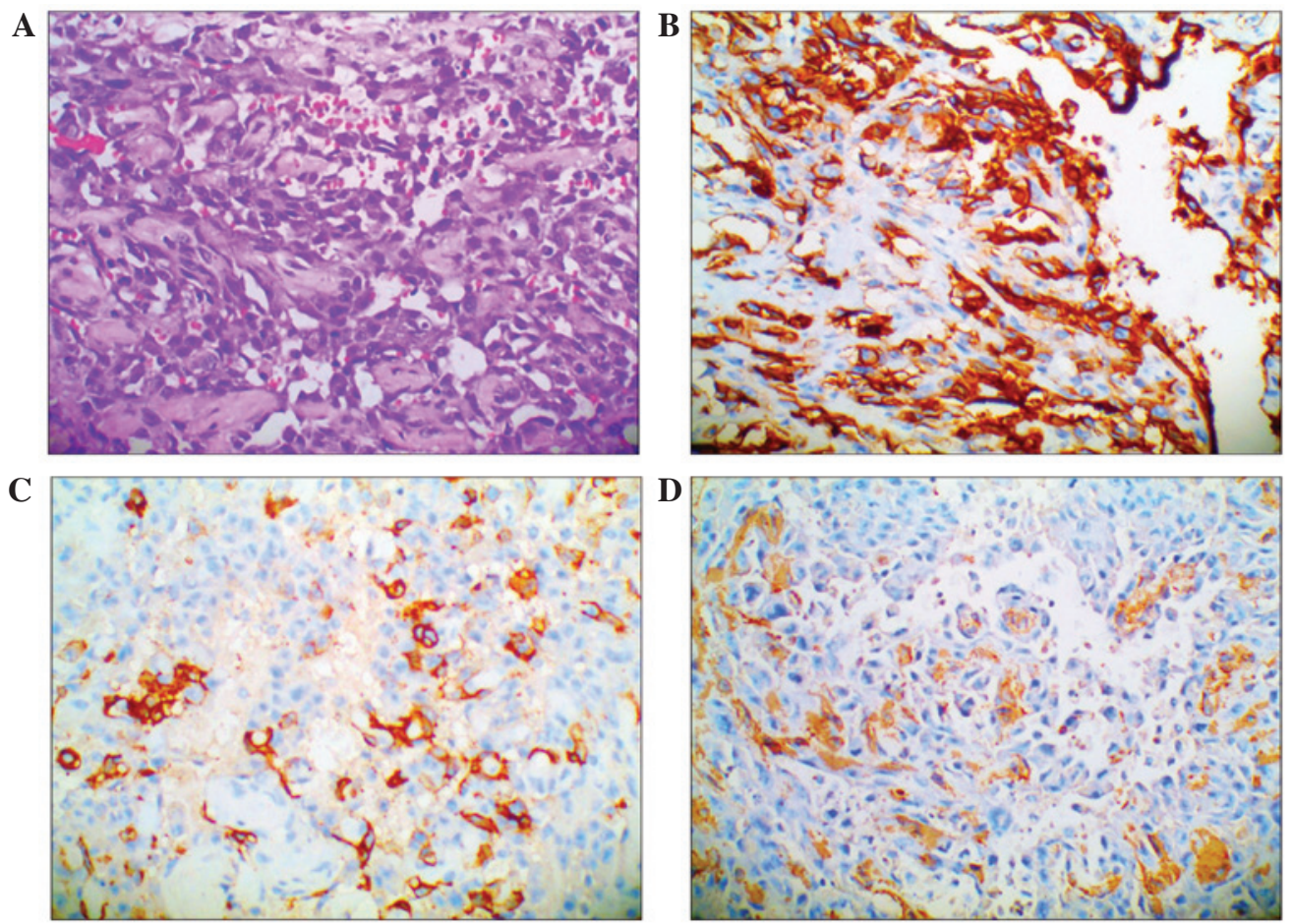

Figure 1. (A) Microscopically, the tumor consisted of spindle-shaped cells with elongated nuclei and displayed staghorn-like vascular channels, mainly of round or ovoid glomus cells (hematoxylin and eosin staining). Immunohistochemical studies demonstrated that the tumor cells expressed (B) CD34; (C) CD31; and (D) $\alpha$-actin. All images: Magnification, x40.
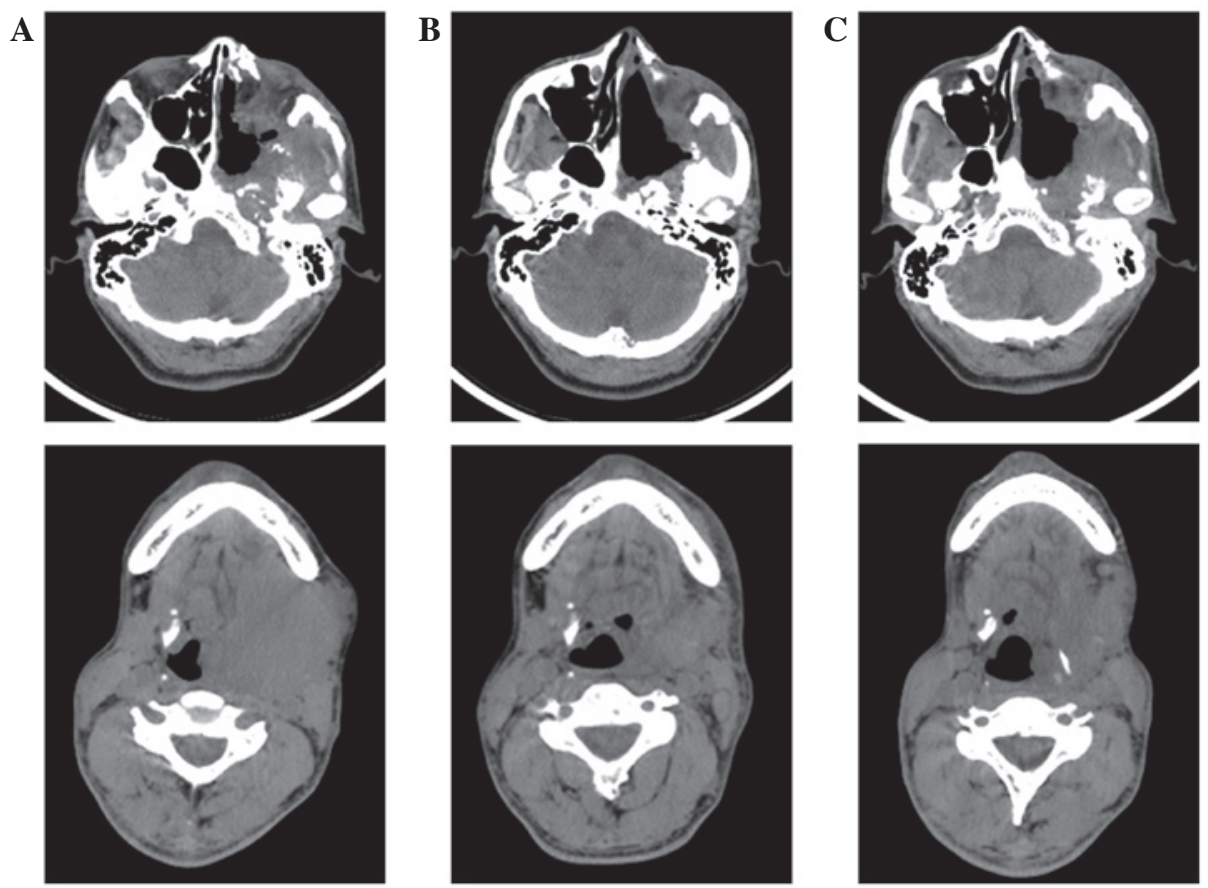

Figure 2. Head (upper panels) and neck (lower panels) CT scans (A) on admission, demonstrating a lamellar high-density shadow in the left temporal lobe and bone destruction in the left sphenoid wing and petrous tip; (B) following 30 cycles of radiotherapy, demonstrating a reduction of masses in the left temporal fossa; and (C) following adjuvant chemotherapy, indicating a further reduction of the masses.

plasm pink). For immunohistochemical assay, endogenous peroxidase activity was blocked with $3 \% \mathrm{H}_{2} \mathrm{O}_{2}$ in methanol for $20 \mathrm{~min}$. Antigen retrieval was performed by microwaving sections in $0.01 \mathrm{M}$ sodium citrate ( $\mathrm{pH}$ 6.0). Non-specific binding was blocked by incubating sections with $5 \%$ bovine serum albumin in phosphate-buffered saline (PBS) for $30 \mathrm{~min}$ at room temperature. Without washing, these sections were incubated with mouse anti-human CD34 monoclonal antibody (catalog no. sc-65261; dilution, 1:500), rabbit anti-human monoclonal CD31 antibody (catalog no. sc-8306; 
$\mathbf{A}$

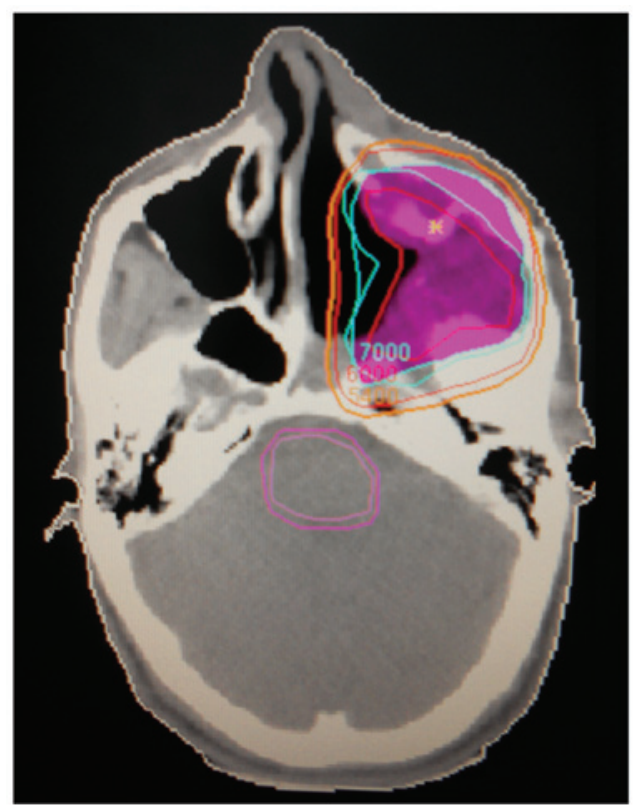

B

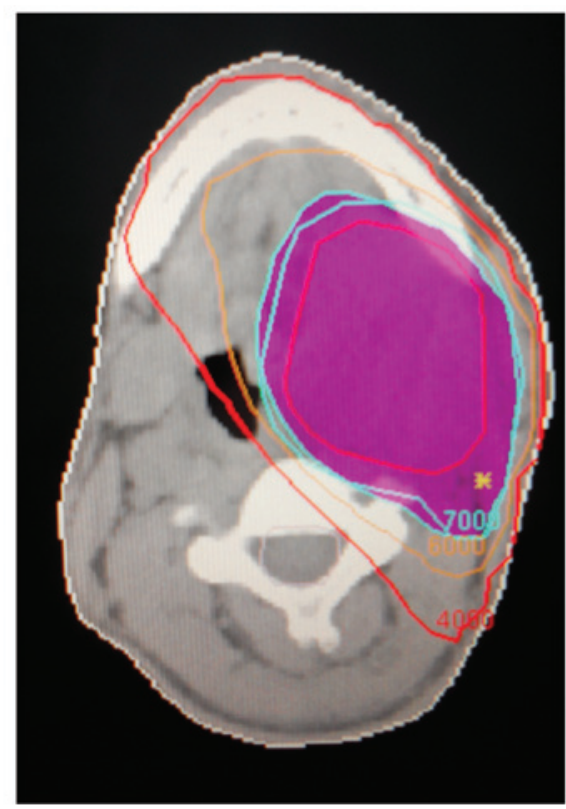

Figure 3. Radiotherapy dosage profile of (A) maxillary sinus layer of head and (B) lymph node layer of neck. All treatment plans (70 Gy, 2 Gy per fraction, 35 fractions) produced adequate target coverage.

dilution, 1:500) or mouse anti-human monoclonal actin antibody (catalog no. sc-58673; dilution, 1:500) in PBS at $4^{\circ} \mathrm{C}$ overnight in a moist box, respectively. After the wash steps, the sections were incubated with corresponding horse radish peroxidase conjugated anti-mouse or anti-rabbit secondary antibodies (Dako Cytomation). The antigen was visualized with substrate chromogen (Dako liquid DAB chromogen; Dako Cytomation). Finally, tissue specimens were stained with Mayer's haematoxylin to discriminate the nucleus from the cytoplasm. Images captured for all sections were acquired using an Olympus CX 31 microscope (Olympus, Tokyo, Japan). Positive cells were indicated by the presence of a distinct brown color in the nuclei or cytoplasm. Normal tissues were used as control tissues, and non-immune IgG was also used as a negative control antibody for immunohistochemical staining. All of the antibodies were purchased from Santa Cruz Biotechnology (Tokyo, Japan).

Under observation, HPC usually consists of spindle-shaped cells with elongated nuclei, and it displays characteristic staghorn-like vascular channels $(6,7)$. In the present study, the tumor cells expressed CD34, CD68(+/-), epithelial membrane antigen, CD31, $\alpha$-actin, desmin, CD99, S-100, B-cell lymphoma-2 (Bcl-2) and Ki-67(30\%), but were negative for creatine kinase (CK) (Fig. 1).

Imaging analysis. On admission, the head and neck CT scan showed a lamellar high-density shadow in the left temporal lobe and bone destruction in the left sphenoid wing and petrous tip. The left sphenoid sinus wall, inside and outside of the board sphenoid wing was absent. Masses were observed in the soft tissues, including the left orbital tissue, temporal fossa, nasopharyngeal and oropharyngeal walls, parapharyngeal space and the masseter gap, indicating multiple recurrent and intracranial invasion of sinonasal HPC. In addition, lymph node enlargement was identified in the neck. The representative CT images are presented in Fig. 2.
Radio- and chemotherapy regimens. As the patient was not able to tolerate surgery, intensity-modulated radiotherapy was adopted. All treatment plans (70 Gy, 2 Gy per fraction, 35 fractions) produced adequate target coverage (ensuring at least $95 \%$ geometrical coverage of the planning target volume). The dose distribution is presented in Fig. 3, in which pink indicates the 70 Gy dose response curve. A CT scan was performed following 30 cycles of radiotherapy and exhibited a reduction of masses in the left temporal fossa and partial restoration of the destructed skull base bones (Fig. 2B). The symptoms were markedly relieved. These findings demonstrated that the tumors responded to radiation therapy.

Following radiation therapy, adjuvant chemotherapy was adopted. Pirarubicin (Shenzhen Main Luck Pharmaceuticals Inc., Shenzhen, China) was administered intravenously on day $1\left(50 \mathrm{mg} / \mathrm{m}^{2}\right)$, and cisplatin was administered intravenously (Qilu Pharmaceutical Co., Ltd., Jinan, China) on days 2-4 $\left(75 \mathrm{mg} / \mathrm{m}^{2}\right)$. As demonstrated in Fig. 2C, a further tumor response was observed following 2 cycles of chemotherapy. No recurrence and metastasis was observed at the 1 year follow-up subsequent to the combined therapy.

This study was approved by the Research Ethics Committee of Tangshan People's Hospital (Tangshan, China). Written informed consent was obtained from the patient. All specimens were handled and anonymized according to ethical and legal standards.

\section{Discussion}

HPC is a relatively indolent neoplasm and commonly behaves in a benign manner, but HPC in the nasal and sinus area often recurs $(1,5)$. Metastasis of sinonasal HPC is rare. The prognosis of sinonasal-type HPC is closely associated with tumor grade, and a higher grade results in a higher mortality rate (5). Although the expression of a number of immunohistochemical markers, such as $\alpha$-actin, CD31 and CD34, has been detected in 
sinonasal HPC tissues $(5,8)$, no specific immunohistochemical markers have been identified. However, staghorn-like vascular channels are considered a histological feature that is specific to sinonasal HPC (5). Although sinonasal HPC is regarded as radioresistant, it has previously been reported that adjuvant radiotherapy may be used for positive surgical margins or recurrent lesions $(1,5)$. In addition, chemotherapy appears to be useful in disseminated $\operatorname{HPC}(4,9,10)$, whilst there is not sufficient evidence of the effectiveness of radiotherapy for sinonasal HPC. It has been previously reported that adriamycin-based chemotherapy was adopted for patient with spleen HPC following simple splenectomy (9). It was recommended that resection coupled with chemotherapy should be performed in cases of resectable recurrence, as it still has a good chance of being curative $(10,11)$. For the first time, pirarubicin and cisplatin were adopted as chemotherapy drugs for the patient with sinonasal HPC and had obvious curative effect.

In the current study, radiotherapy followed by chemotherapy (pirarubicin combined with cisplatin) demonstrated positive efficacy. Therefore, for cases of multiple recurrent and invasive sinonasal HPC, combined treatment of radiotherapy and chemotherapy is recommended, but additional cases are required to evaluate the clinical efficacy and impact on survival.

\section{References}

1. Billings KR, Fu YS, Calcaterra TC and Sercarz JA: Hemangiopericytoma of the head and neck. Am J Otolaryngol 21: $238-243,2000$.
2. Terada T and Kato T: Sinonasal-type hemangiopericytoma of the nasal cavity and paranasal sinus. Int J Clin Oncol 17: $169-173,2012$

3. Duman FU, Ayhan S, Işısă A, Eskıızmır G and Tarhan S: Sinonasal-type haemangiopericytoma: A case report. Turk Patoloji Derg: Mar 18, 2014 (Epub ahead of print) (In Turkish).

4. Dahodwala MQ, Husain Q, Kanumuri VV, Choudhry OJ, Liu JK, and Eloy JA. Management of sinonasal hemangiopericytomas: a systematic review. Int Forum Allergy Rhinol. 2013;3(7):581-7.

5. Thompson LD, Miettinen M and Wenig BM: Sinonasal-type hemangiopericytoma: a clinicopathologic and immunophenotypic analysis of 104 cases showing perivascular myoid differentiation. Am J Surg Patho1 27: 737-749, 2003.

6. Fletcher CD1. Distinctive soft tissue tumors of the head and neck. Mod Pathol. 2002;15(3):324-30.

7. Agaimy A1, Barthelmeß S, Geddert H, Boltze C, Moskalev EA, Koch M, Wiemann S, Hartmann A, Haller F. Phenotypical and molecular distinctness of sinonasal haemangiopericytoma compared to solitary fibrous tumour of the sinonasal tract. Histopathology. 2014;65(5):667-73.

8. Yokoi H, Arakawa A, Kuribayashi K, Inoshita A, Haruyama T, and Ikeda K: An immunohistochemical study of sinonasal hemangiopericytoma. Auris Nasus Larynx 38: 743-746, 2011.

9. Illuminati G, Pizzardi G, Calio F, Pacilè MA, Carboni F, Palumbo P and Vietri F: Hemangiopericytoma of the spleen. Int J Surg 15: 6-10, 2015.

10. Iwamuro M, Nakamura $\mathrm{S}$, Shiraha $\mathrm{H}$, et al: A case of primary intracranial hemangiopericytoma with hepatic metastases: successful treatment with radiofrequency ablation and transcatheter arterial chemoembolization. Clin J Gastroenterol 2: 30-35, 2009.

11. Maria PS, Mauri M and Carmignani L: A unique cause of hemoperitoneum: spontaneous rupture of a splenic hemangiopericytoma. Int J Emerg Med 4: 13, 2011. 